\title{
A COMPARATIVE STUDY OF INFUSION DEXMEDETOMIDINE AND INFUSION MAGNESIUM SULPHATE ON ATTENUATION OF BLOOD PRESSURE SURGE IN LAPAROSCOPIC SURGERY UNDER GENERAL ANAESTHESIA
}

\author{
Suchismita Mallick1, Sarbari Sawika², Siddhartha Chakraborty33, Subhasish Ghosh ${ }^{4}$, Rajat Choudhuri ${ }^{5}$ \\ ${ }^{1}$ Assistant Professor, Department of Anaesthesiology and Critical Care, Institute of Post Graduate Medical Education and Research, \\ Kolkata, West Bengal, India. \\ ${ }^{2}$ Associate Professor, Department of Anaesthesiology and Critical Care, Institute of Post Graduate Medical Education and Research, \\ Kolkata, West Bengal, India. \\ ${ }^{3}$ Senior Resident, Department of Anaesthesiology and Critical Care, Institute of Post Graduate Medical Education and Research, \\ Kolkata, West Bengal, India. \\ ${ }^{4}$ Senior Resident, Department of Anaesthesiology and Critical Care, Institute of Post Graduate Medical Education and Research, \\ Kolkata, West Bengal, India. \\ ${ }^{5}$ Associate Professor, Department of Anaesthesiology and Critical Care, Institute of Post Graduate Medical Education and Research, \\ Kolkata, West Bengal, India.
}

\begin{abstract}
BACKGROUND
ABSTRACT

The haemodynamic surge in laparoscopic surgery following carbon dioxide insufflations can be attenuated by various drugs. Dexmedetomidine, a selective-alpha-agonist depresses central sympathetic outflow producing sedation, decreased analgesic requirement, is approved by USFDA for mechanically ventilated patients in ICU for a period of < 24 hours. One of its "off label" uses is attenuation of Hemodynamic changes in laparoscopic surgery. Parenteral magnesium sulphate has been used as an antiarrhythmic and antiseizure drug in preeclampsia. It also has analgesic action on NMDA receptor, inhibits catecholamine release from peripheral nociceptors and attenuates vasopressor mediated vasoconstriction. A study was conducted to assess and compare the effects of these drugs. We wanted to study the relative efficacy of infusions of dexmedetomidine \& magnesium sulphate on attenuation of hemodynamic responses in laparoscopic surgery under general anaesthesia.
\end{abstract}

\section{METHODS}

A prospective, double blind, randomized, comparative study was conducted on a total of 105 patients in a tertiary care teaching hospital. The three groups ( $\mathrm{n}=35$ ) were: Control (Group-C), Dexmedetomidine (Group-D), and Magnesium sulphate (Group-M).

\section{RESULTS}

Heart rate, systolic blood pressure, diastolic blood pressure, mean arterial pressure were significantly lower with Dexmedetomidine group than Magnesium sulphate group at all intervals $(\mathrm{p}<0.05)$ The MgSo4 group however showed significantly $(\mathrm{p}<0.05)$ better haemodynamic control at 30 minutes compared to the control group.

\section{CONCLUSIONS}

Intravenous infusion with Dexmedetomidine for laparoscopic surgery under general anaesthesia is more effective than Magnesium sulphate in attenuating the cardiovascular effects of pneumoperitoneum.

HOW TO CITE THIS ARTICLE: Mallick S, Sawika S, Chakraborty S, et al. A comparative study of infusion dexmedetomidine and infusion magnesium sulphate on attenuation of blood pressure surge in laparoscopic surgery under general anaesthesia. J. Evolution Med. Dent. Sci. 2019;8(21):1695-1699, DOI: 10.14260/jemds/2019/373

\section{BACKGROUND}

In order to reduce morbidity, mortality and hospital stay with consequent reductions in health care cost, laparoscopic procedures are the preferred choice of surgical approach. Hemodynamic changes in laparoscopic surgery are a major concern for anaesthesiologist. The two main factors responsible for the hemodynamic variations are raised $\mathrm{PaCO}_{2}$ and intra-abdominal pressure (IAP).

'Financial or Other Competing Interest': None.

Submission 28-01-2019, Peer Review 10-05-2019,

Acceptance 16-05-2019, Published 27-05-2019.

Corresponding Author:

Dr. Sarbari Swaika,

Associate Professor,

Department of Anaesthesiology and Critical Care,

Institute of Post Graduate Medical Education and Research,

Kolkata, West Bengal, India.

E-mail: dr.s.swaika@gmail.com

DOI: $10.14260 /$ jemds $/ 2019 / 373$

(c) $(i)$
Increase in IAP leads to increase in mean arterial resistance, ${ }^{1,2,3}$ myocardial oxygen demand, leading to complications such as myocardial ischemia, cerebral haemorrhage. The neuroendocrine factors also contribute to the hemodynamic changes induced by $\mathrm{CO}_{2}$ pneumoperitoneum.

An increase in intra-abdominal pressure more than 10 mmHg and patient positioning inflicts significant changes including decreased thoraco-pulmonary compliance $30 \%$ to $50 \%$ ), increased systemic and pulmonary vascular resistance (SVR and PVR) and caval compression leading to decreased venous return, decreases cardiac output (10\% to 30\%). These factors can precipitate an adverse cardiac event in patients with pre-existing cardiovascular diseases.

Various pharmacological agents are being used to attenuate the hemodynamic response to pneumoperitoneum. These include Beta-blockers like Atenolol, Esmolol,4 calcium channel blocker, ${ }^{5} \alpha_{2}$-adrenergic receptors agonists clonidine, ${ }^{6} \quad$ dexmedetomidine $^{7} \quad$ Opioids-Fentanyl, Remifentanil; Vasodilators-Nitroglycerin, Nicardipine. Many 
clinical trials on healthy individuals have shown that $\alpha 2$ adrenergic receptor agonists, magnesium sulphate ${ }^{8}$ are also effective in controlling these hemodynamic variations. In this study we have compared the variations in heart rate (HR), blood pressure (BP), mean Arterial Pressure (MAP) following intravenously administered magnesium sulphate with dexmedetomidine as premedication during Laparoscopic surgery under general anaesthesia.

\section{METHODS}

A prospective, double blind, randomized, comparative study was conducted on a total of 105 patients at Department of Anaesthesiology, IPGME\&R, Kolkata, from January 2015 to October 2016. Patients were randomly assigned to one of the following three groups using a computer generated randomnumber table. It was estimated that 35 subjects would be required per group in order to detect the difference of 10 $\mathrm{mmHg}$ in between the groups with $10 \%$ power and $5 \%$ probability of type 1 error. This calculation assumes standard deviation of $10 \mathrm{mmHg}$ in mean arterial blood pressure Variation in mean arterial blood pressure (Between baseline and post insufflation) was. Sample size calculation was done with nMaster 2.0 software (Dept. of Biostatistics, Christian Medical College, Vellore)

Control Group $(n=35)$ was given infusion of $0.9 \%$ NS (Group-C).

Dexmedetomidine Group $(n=35)$ will be given Dexmedetomidine infusion at a dose of $1 \mathrm{mcg}$./ $/ \mathrm{kg}$ over 10 min. followed by $0.5 \mathrm{mcg} / \mathrm{kg} /$ hour (Group-D). (200 mcg of Dexmedetomidine will be dissolve in $0.9 \%$ NS up to $50 \mathrm{ml}$, each $\mathrm{ml}$ constitutes of $4 \mathrm{mcg}$ Dexmedetomidine.)

Magnesium sulphate Group ( $n=35)$ will be given infusion of magnesium sulphate at a dose of $30 \mathrm{mg} / \mathrm{kg}$. over $10 \mathrm{~min}$ followed by $10 \mathrm{mg} / \mathrm{kg} /$ hour (Group-M) (12 ml 50\% magnesium sulphate will be dissolved in $0.9 \%$ NS up to $50 \mathrm{ml}$, each $\mathrm{ml}$ constitutes of $120 \mathrm{mg}$ magnesium sulphate.)

The patients were assigned to each group according to random number table and the anaesthesiologist prepared the drugs in $50 \mathrm{ml}$ infusions and another anaesthesia personnel unaware of the drugs used administered them and recorded the data. All the infusions were colourless. Thus, the double blinding of the study was done.

Patients of either sex, aged between 18-60 yrs., body weight ranging from $50-70 \mathrm{~kg}$, belonging to ASA physical group I posted for elective abdominal laparoscopic surgery under general anaesthesia in surgical main OT of IPGME\&R, Kolkata.

The patients with refusal, emergency surgery, allergy to trial drugs, history of drug and alcohol abuse, patients on cardioactive drugs like alpha 2 agonist, methyldopa, beta blockers, calcium channel blocker, ACE inhibitors, anticipated difficult intubation (Mallampati grade III or IV), pregnancy and patients with severe systematic disorders like diabetic, musculoskeletal and neurological disease were excluded from the study.

On the day of surgery, 8-hour preoperative fasting guideline was maintained. Tab pantoprazole $40 \mathrm{mg}$ and tab midazolam $7.5 \mathrm{mg}$ at bedtime was given. Pre-anaesthetic evaluation was performed in each patient including detailed history taking, thorough clinical examination, assessment of spine, airway examination and usual haematological investigation, Chest $\mathrm{X}$ ray \& ECG. After arrival of the patient in operation theatre, monitors (ECG leads, blood pressure cuff and pulse oximeter probe) were attached. Anaesthesia machine, airway equipment, Suction apparatus drugs for resuscitation and general anaesthesia were kept ready in hand before starting the procedure. The patients were monitored by NIBP, continuous ECG, pulse oximetry and $\mathrm{EtCO}_{2}$ throughout the surgery. In supine position after premedication with $10 \mathrm{mcg} / \mathrm{kg}$ glycopyrrolate and fentanyl 2 $\mu \mathrm{g} / \mathrm{kg}$ iv, patient was preoxygenated with $100 \% \mathrm{O}_{2}$ for 3 minutes, general anaesthesia was induced with IV propofol 2 $3 \mathrm{mg} / \mathrm{kg}$ followed by atracurium $0.5 \mathrm{mg} / \mathrm{kg}$ iv to facilitate endotracheal intubation. Tracheal intubation was done and confirmed by bilateral chest auscultation and EtCo2 monitoring. Anaesthesia was maintained with nitrous oxide and oxygen, Muscle relaxation with intermittent dose of atracurium $0.1 \mathrm{mg} / \mathrm{kg}$ IV. Heart rate, systolic blood pressure (SBP), diastolic blood pressure (DBP) mean arterial pressure (MAP), respiratory rate, $\mathrm{SPO}_{2}, \mathrm{ETCO}_{2}$ values were recorded. Controlled ventilation was done with Bain's coaxial breathing system to maintain an $\mathrm{EtCO}_{2}$ level between $35-40 \mathrm{~mm}$ of $\mathrm{Hg}$. Any hypotension (mean arterial pressure lower than $20 \%$ of baseline) or bradycardia (heart rate $<50 / \mathrm{min}$ ) incidents was treated with phenylephrine 50-100 microgram or atropine $0.6 \mathrm{mg}$ increments. A decrease in $\mathrm{Sp}_{02}$ to $<90 \%$ was defined as hypoxia and was to be treated accordingly. After completion of surgery, neuromuscular block was reversed with appropriate dose of neostigmine and glycopyrrolate. Patients were then transferred to recovery room.

For statistical analysis $\mathrm{P}$ value $<0.05$ will be considered significant. Continuous variables like age, weight, Heart Rate, SBP, DBP, MAP, RPP, SPO2 and ETCO2 are expressed as Mean \pm Standard Deviation and compared across the 3 group.

Software used were statistica version 6 [Tulsa, Oklahoma: StatSoft Inc., 2001], SPSS Statistics version 17 [Illinois, Chicago: SPSS Inc., 2008], Graph Pad Prism version 5 [San Diego, California: Graph Pad Software Inc., 2007]. All numerical variables were normally distributed by Kolmogorov-Smirnoff goodness-of-fit test. Categorical variables like sex, ASA grade and ECG outcomes were expressed as number of patients and percentage of patients and compared across the 3 groups using Pearson's Chi Square test for Independence of Attributes. Comparison of numerical variables between Groups 1, 2 and 3 was done with One-way ANOVA and Post-hoc comparisons by Tukey's test. An alpha level of $5 \%$ was considered as significant.

\section{RESULTS}

\section{Comparison of Mean Heart Rate Among the Groups}

Figure 1 (CHART) show the comparison of heart rate among three groups. Heart rate is normally distributed by Kolmogorov-Smirnoff goodness-of-fit test. Applying the One way ANOVA test, it is found that there is no statistically significant difference in HR among the groups ( $\mathrm{p}$ value > 0.05 ) at baseline, $0 \mathrm{~min}$, and up to $5 \mathrm{~min}$. After 10, 20, 30, and up to 40 minutes of pneumoperitoneum, H.R. was found to be significantly lower in dexmedetomidine than magnesium sulphate and control groups ( $\mathrm{p}$ value $<0.05$ ). 

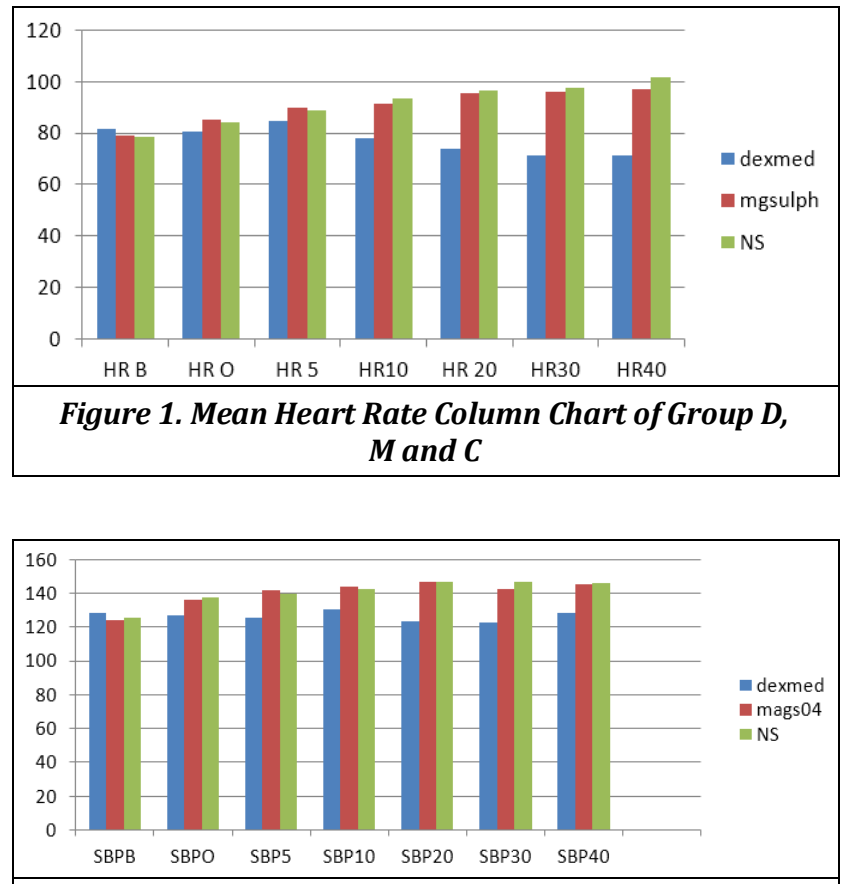

Figure 2. SBP Variation Column Chart of Group D, M and C

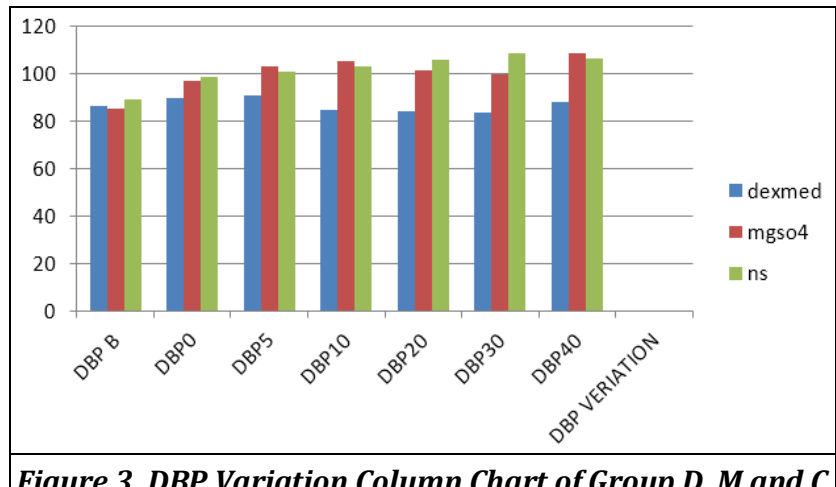

Figure 3. DBP Variation Column Chart of Group D, M and C

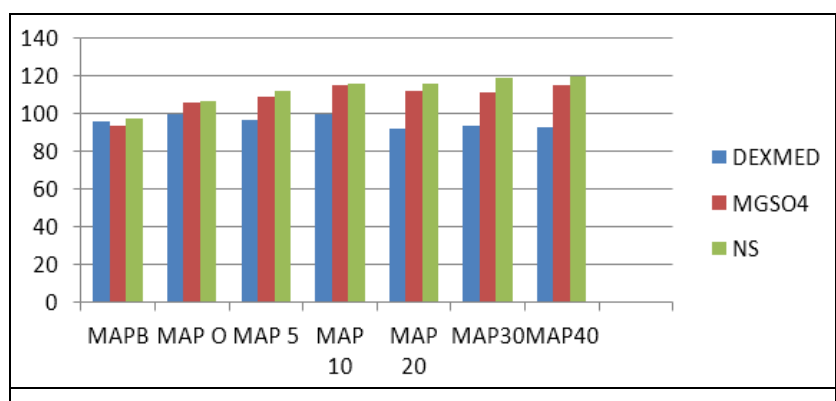

Figure 4. MAP Variation Column Chart of Group D, M and C

\section{Comparison of Systolic Blood Pressure Among the Groups}

Figure 2(column chart), show the comparison of systolic blood pressure among three groups. Variable is normally distributed by Kolmogorov-Smirnoff goodness-of fit test. Applying the One-way ANOVA test it is found that there is no statistically significant difference ( $p$ value $>0.05$ ) among the groups at baseline systolic blood pressure ( $p$ value 0.321 ). Then after starting and up to 40 mins of pneumoperitoneum SBP was significantly lower with dexmedetomidine than with magnesium sulphate and control groups ( $p$ value $<0.05$ ).
Comparison of Diastolic Blood Pressure Among the Groups

Figure 3 (column chart) show the comparison of diastolic blood pressure among three groups. Variable (diastolic blood pressure) is normally distributed by Kolmogorov-Smirnoff goodness-of fit test. Applying the One-way ANOVA test it is found that there is no statistically significant difference ( $p$ value $>0.05$ ) among the groups at baseline diastolic blood pressure. ( $p$ 0.322).Then after starting and upto 40 mins of pneumoperitoneum DBP was significantly lower with dexmedetomidine than with magnesium sulphate and control groups ( $p$ value $<0.05$ ) Post-hoc comparisons (Following ANOVA) by Tukey's test, found that there is significant difference $(\mathrm{p}<0.05)$ of DBP at $30 \mathrm{~min}$. of pneumoperitoneum.

\section{MAP Variation Among the Groups}

Figure 4 show the comparison of mean blood pressure among three groups. Variable (Mean blood pressure) is normally distributed by Kolmogorov-Smirnoff goodness-of-fit test. Applying the One-way ANOVA test, it was found that there was no statistically significant difference ( $p$ value $>0.05$ ) among the groups at baseline mean blood pressure $(\mathrm{p}$ value 0.331).Then after starting and up to 40 mins of pneumoperitoneum, MAP significantly lower with dexmedetomidine than with magnesium sulphate and control groups ( $\mathrm{p}$ value $<$ 0.05).

\section{DISCUSSION}

This study evaluated the efficacy of intravenous premedication with dexmedetomidine versus magnesium sulphate on attenuation of the cardiovascular effects due to pneumoperitoneum during laparoscopic surgery under general anaesthesia. Since the advent of laparoscopy in the early 1970s, anaesthesia for laparoscopy is continuously being improvised with the search for an ideal anaesthetic drug to attenuate the hemodynamic responses due to carbon dioxide $\left(\mathrm{CO}_{2}\right)$ pneumoperitoneum. With an alpha error of $5 \%$ and $p$ value of 0.05 , the sample size was decided to be 35 in each of the three groups. There was no significant difference between the demographic profiles of the three groups.

The baseline heart rates were comparable in all the groups. H.R. is significantly lower in dexmedetomidine than magnesium sulphate and control groups ( $\mathrm{p}$ value is less than 0.05). This is because in our study we have administered dexmedetomidine as a bolus dose and maintenance dose. The lack of significant difference at baseline, $0 \mathrm{~min}$, and upto 5 min is probably due to predominant effect of anaesthetic agents administered at the time of induction.

Our study correlated with the study done by Nand Kishore Kalra et al, ${ }^{9}$ a study in which administration of magnesium sulphate and clonidine was done for attenuation of hemodynamic responses to pneumoperitoneum. The authors concluded that Clonidine $(1.5 \mathrm{mcg} / \mathrm{kg})$ blunts hemodynamic responses to pneumoperitoneum more effectively than magnesium sulphate $(50 \mathrm{mg} / \mathrm{Kg})$ used 15 mins before pneumoperitoneum. In this study also dexmedetomidine it was observed that dexmedetomidine group was more effective in the attenuation of haemodynamic surge than magnesium sulphate group. 
Magnesium sulphate group was observed to have marginally lower clinical values of SBP, MAP but it was statistically insignificant compared to the control group. The only significant finding with magnesium sulphate group was found in the reading of DBP by Post-hoc comparisons (Following ANOVA) by Tukey's test at $30 \mathrm{~min}$. of pneumoperitoneum. A significant difference ( $p$ value is less than 0.05 ) was observed in this value. Out of 35 cases, 3 cases of groupM slightly delayed neuromuscular recovery were noted. Kalra et al. assessed which of magnesium or clonidine attenuates hemodynamic stress response to pneumoperitoneum better and found no significant difference between magnesium and clonidine groups given in dose of $1 \mu \mathrm{g} / \mathrm{kg}$. However, the attenuation of the surge was observed to be better in patients receiving clonidine in dose of $1.5 \mu \mathrm{g} / \mathrm{kg}$. Godhki et al used Dexmedetomidine $(1 \mathrm{mcg} / \mathrm{kg})$ over $10 \mathrm{~min}, 15 \mathrm{~min}$. before induction and infusion $0.2 \mathrm{mcg} / \mathrm{kg} / \mathrm{hour}$ in laparoscopic surgery. They found that Dexmedetomidine can be safely used in laparoscopic surgery and no incidence of awareness was recorded. ${ }^{10}$ Smania et al found that dexmedetomidine efficiently blocks the hemodynamic responses to nociceptive stimuli when combined with inhaled isoflurane for anaesthesia of children submitted to laparoscopic video appendectomy. ${ }^{11}$ The effect of dexmedetomidine on haemodynamics is due to decrease of sympathetic outflow from the locus ceruleus. Its sympatholytic effect leads to decrease of mean arterial blood pressure (MAP) and heart rate (HR) by reducing norepinephrine release. Its analgesic actions are mediated by release of substance $\mathrm{P}$ from the dorsal horn of the spinal cord. Gourishankar Reddy Manne et $\mathrm{al}^{12}$, in 2014 compared the effects of low dose dexmedetomidine infusion $(0.2 \mathrm{mcg} / \mathrm{Kg} / \mathrm{h}$ and $0.4 \mathrm{mcg} / \mathrm{Kg} / \mathrm{h}$ started 15 minutes before induction) with normal saline as control, on hemodynamic stress response, sedation and postoperative analgesia requirement in 60 patients. They reported that dexmedetomidine infusion in the dose of 0.4 $\mathrm{mcg} / \mathrm{kg} / \mathrm{h}^{13}$ effectively attenuates haemodynamic stress response during laparoscopic surgery with reduction in postoperative analgesic requirements. We used dexmedetomidine of $1 \mathrm{mcg} / \mathrm{kg}$ body weight over 10 minutes, 15 min before induction of pneumoperitoneum, followed by 0.5 $\mathrm{mcg} / \mathrm{kg} /$ hour. In our study also similar effects with dexmedetomidine were observed. Vaishali Waindeskar et al ${ }^{14}$ assessed the role of dexmedetomidine as an anaesthetic adjuvant in laparoscopic surgeries. 60 patients were divided into two groups, the control group was given normal saline. Dose of dexmedetomidine used was $1 \mathrm{mcg} / \mathrm{Kg}$ as loading dose and maintained with $0.2 \mathrm{mcg} / \mathrm{Kg} / \mathrm{h}$ infusion. They reported that the fall in mean heart rate and mean systolic blood pressure with respect to dexmedetomidine was significant compared to the control group. The same loading dose and maintenance with $0.3 \mathrm{mcg} / \mathrm{kg} /$ hour infusion was used in this study too with similar effects. Jee et al. ${ }^{15}$ administered magnesium sulphate $50 \mathrm{mg} / \mathrm{kg}$ over 2-3 min, before pneumoperitoneum in patients undergoing laparoscopic cholecystectomy and found that it effectively attenuated the haemodynamic effects of pneumoperitoneum without any episode of severe hypotension or bradycardia. In this study also, although no significant difference was observed between group $\mathrm{M}$ and $\mathrm{C}$, the clinical values of SBP, HR were lower in group $M$ than group $C$ and no severe hypotensive episode was noted in group $M$. This may be due to the fact that plasma catecholamine and vasopressin concentrations are significantly lowered by magnesium and hence magnesium sulphate effectively blunted the sympathoadrenal hemodynamic stress responses to pneumoperitoneum. The reduction in blood pressure could be attributed to vasodilatory effect of magnesium sulphate. Our results show that in group $M$, both SBP and DBP increased abruptly after pneumoperitoneum compared to group D and this increase was sustained throughout the procedure and haemodynamic response to pneumoperitoneum in patients of group D was attenuated as shown by significant reduction of SBP and DBP.

Other studies have suggested that magnesium can inhibit catecholamine release in vitro ${ }^{16}$ and in vivo. Serum magnesium concentrations of $2-4$ mmols/l are required to exert these effects. Jee et al. found that magnesium sulphate bolus of $50 \mathrm{mg} / \mathrm{kg}$ before pneumoperitoneum increased serum magnesium concentrations to this range. Like catecholamines, vasopressin also contributes to hemodynamic changes induced by pneumoperitoneum. Vasopressin concentration increases when intra-abdominal pressure compresses abdominal capacitance vessels, and this reduces intra-thoracic blood volume and a decrease in venous return. Vasodilatory effects of magnesium sulphate lead to dilatation of abdominal vessels which might prevent vasopressin release. Further studies are however needed to determine precise mechanism by which magnesium reduces vasopressin concentration.

As a further support for efficacy of both drugs, Bryskin and Weldon ${ }^{17}$ used a combination of dexmedetomidine and magnesium sulphate for hemodynamic control during laparoscopic resection of phaeochromocytoma and reported that cardiovascular stability was achieved. However, other comparative studies provided evidence for their superiority to other drugs used for the same target, where Salman et al.18 compared dexmedetomidine with remifentanil in desfluranebased ambulatory gynaecologic laparoscopic surgery and demonstrated that dexmedetomidine infusion causes a relatively slow recovery with reduced postoperative nausea, vomiting, and analgesic requirements, and similar haemodynamics compared to remifentanil, and may be an alternative to remifentanil in ambulatory anesthesia. ${ }^{19}$

\section{CONCLUSIONS}

Intravenous premedication with dexmedetomidine infusion for laparoscopic surgery under general anaesthesia is significantly more effective than Magnesium sulphate in attenuating the cardiovascular effects of pneumoperitoneum and therefore beneficial for the patient. This study involved ASA physical status Class I patients only. Future studies involving ASA physical status Class II, III and IV patients, e.g. patients with uncontrolled hypertension and/or uncontrolled diabetes mellitus and increased number of patients can be done.

\section{REFERENCES}

[1] Johannsen G, Andersen M, Juhl B. The effects of general anaesthesia on the haemodynamic events during laparoscopy with CO2-insufflation. Acts anaesthesol Scand 1989;33(2):132-6. 
[2] Westerband A, Van De Water J, Amzallag M, et al. Cardiovascular changes during laparoscopic cholesystectomy. Surg Gynecol Obstet 1992;175(6):535-8.

[3] O'Malley C, Cunningham AJ. Physiology changes during laparoscopy. Anesthesiol Clin North Am 2001;19(1):119.

[4] Dyson A, Isaac PA, Pennant JH, et al. Esmolol attenuates cardiovascular responses to extubation. Anesth Analg 1990;71(6):675-8.

[5] Kovac AL, Masiongale A. Comparison of nicardipine versus esmolol in attenuating the hemodynamic responses to anesthesia emergence and extubation. J Cardiothorac Vasc Anesth 2007;21(1):45-50.

[6] Taittonen M, Kirvelä O, Aantaa R, et al. Cardiovascular and metabolic responses to clonidine and midazolam premedication. Eur J Anaesthesiol 1997;14(2):190-6.

[7] Basar H, Akpinar S, Doganei N, et al. The effect of preanaesthetic, single dose Dexmedetomidine on induction, hemodynamic and cardiovascular parameters. J Clin Anaesth 2008;20(6):431-6.

[8] Elsharnouby NM, Elsharnouby MM. Magnesium sulphate as a technique of hypotensive anaesthesia. $\mathrm{Br}$ J Anaesth 2006;96(6):727-31.

[9] Kalra NK, Verma A, Agarwal A, et al. Comparative study of intravenously administered clonidine and Magnesium Sulphate on hemodynamic responses in laparoscopic surgery. J Anaesthesiol Clin Pharmacol 2011;23(3):344-8.

[10] Ghodki PS, Thombre SK, Sardesai SP, et al. Dexmedetomidine as an anesthetic adjuvant in laparoscopic surgery: an observational study using entrophy monitoring. J Anaesthesiol Clin Pharmacol 2012;28(3)334-8.

[11] Smania MC, Piva JP, Garcia PC. Dexmedetomidine in anesthesia of children submitted to videolaparoscopic appendectomy: a double-blind, randomized and placebo-controlled study. Rev Assoc Med Bras (1992) 2008;54(4):308-13.
[12] Manne GR, Upadhyay MR, Swadia V. Effects of low dose dexmedetomidine infusion on haemodynamic stress response, sedation and post-operative analgesia requirement in patients undergoing laparoscopic cholecystectomy. Indian J Anaesth 2014;58(6):726-31.

[13] Easley RB, Brady KM, Tobias JD. Dexmedetomidine for the treatment of postanaesthesia shivering in children. Paediatric Anaesthesia 2007;17(4):341-6.

[14] Waindeskar V, Khan M, Agarwal S, et al. Role of Dexmedetomidine as an anesthetic adjuvant in laparoscopic surgery. People's Journal of Scientific Research 2015;8(2):46-50.

[15] Jee D, Lee D, Yun $S$, et al. Magnesium sulphate attenuates arterial pressure increase during laparoscopic cholecystectomy. $\mathrm{Br} \quad \mathrm{J}$ Anaesth 2009;103(4):484-9.

[16] Herroeder S, Schönherr ME, De Hert SG, et al. Magnesium-essentials for anesthesiologists. Anesthesiology 2011;114(4):971-93.

[17] Bryskin R, Weldon BC. Dexmedetomidine and Magnesium Sulfate in the perioperative management of a child undergoing laparoscopic resection of bilateral pheochromocytomas. Journal of Clinical Anesthesia 2010;22(2):126-9.

[18] Salman N, Uzun S, Coskun F, et al. Dexmedetomidine as a substitute for remifentanil in ambulatory gynecologic laparoscopic surgery. Saudi Medical Journal 2009;30(1):77-81.

[19] Peden CJ, Cloote AH, Stratford N, et al. The effect of intravenous dexmedetomidine premedication on the dose requirement of propofol to induce loss of consciousness in patients receiving alfentanil. Anaesthesia 2001;56(5):408-13. 